\title{
Realistic confinement potential for a square-patterned two-dimensional semiconductor quantum dot and its approximated circular counterpart
}

\author{
Orion Ciftja
}

\begin{abstract}
Two-dimensional semiconductor quantum-dot systems are typical nanoscale structures in which a few number of electrons is confined in a small region of space by applying external electric gate potentials. While the detailed form of the confining potential depends on the specific experimental setup, the parabolic confinement model has commonly been used because of its simplicity. Clearly, on those instances in which the experimental setup involves placement of gate potentials with sharp geometric features, the area depleted of electrons; thus, the quantum-dot region cannot be considered circular. If, for simplicity, we consider the confinement region of the electrons as square in shape, then an accurate calculation of the properties of such square-patterned quantum dot should be made using a realistic confinement potential originating from that particular configuration. We calculated exactly such a confinement potential for a square quantum dot. The particular analytic form of this realistic potential is complicated given its dependence on the two-dimensional position coordinates, rather than simply the distance from the center of the quantum dot. In this work, we choose to substitute the realistic confinement potential for a square-patterned quantum dot with an approximated circular symmetric potential. We assess the quality of this approximation and discuss instances in which one can reliably use the approximated simplified potential instead of the computationally unyielding exact one.
\end{abstract}

Keywords: Semiconductor quantum dots, Confinement potential, Coulomb interaction

\section{Background}

Two-dimensional (2D) semiconductor quantum-dot systems are typical nanoscale structures in which a few number of electrons is confined in a small region of space by applying external electric gate potentials [1,2]. Such systems represent one important step towards future generations of sophisticated nanoscale device technologies $[3,4]$ and also are important from a fundamental theoretical point of view since they represent an ideal setting to investigate the interplay between many quantum effects that otherwise are not readily available [5-13]. Confinement and electron correlations manifest themselves in many interesting phenomena including signatures of the very interesting physics of the fractional quantum Hall

Correspondence: ogciftja@pvamu.edu

Department of Physics, Prairie View A\&M University, Prairie View, TX, 77446, USA effect phases [14-19] if a high perpendicular magnetic field is applied. To some extent, small 2D semiconductor quantum dots mirror naturally occurring atomic systems [20-22] with the important difference of the confinement potential which is artificially created. Freedom to choose the confinement potential is extremely desirable since it implies high tunability of physical properties using standard experimental methods. While the detailed form of the confining potential depends on the specific experimental setup, the parabolic confinement model has commonly been used because of its simplicity [23-26]. Despite the variety of confinement potentials with both infinite and finite range, most of these choices share the property of being isotropic in the 2D spatial directions $[27,28]$. While these confinement models generally work well, there are situations in which the experimental setup in a $2 \mathrm{D}$ semiconductor quantum dot involves asymmetric 
application of several gate potentials with sharp geometric features.

In instances in which the experimental setup involves placement of gate potentials with sharp geometric features, the area depleted of electrons, thus the quantum dot region, is not circular. If, for simplicity, we consider the confinement region of the electrons as square in shape, then an accurate calculation of the properties of such square-patterned quantum dot should be made by using a realistic confinement potential originating from that particular configuration. In this work, we consider the confining region of the electrons to be precisely a square and, thus, calculate exactly the confinement potential originating from this geometry. We find out that the particular analytic form of this confinement potential is quite complicated given the dependence of the potential on the separate $x$ and $y$ coordinates. Given that the realistic confinement potential for a square-patterned 2D semiconductor quantum dot is complicated, it makes sense to approximate it with simpler forms that possess circular symmetry if the loss of accuracy is not prohibitive. In this work, we study a circular confinement potential that is a good approximation to the resulting squarepatterned one. We assess the quality of this approximation and discuss instances in which one can reliably use the approximated simplified circular potential instead of the computationally unyielding exact one.

\section{Methods}

\section{Confinement potential due to a square patterned two-dimensional semiconductor quantum dot}

Depending on the placement of the gate potentials in a 2D semiconductor quantum dot, electrons might get confined in a non-circular region with sharp geometric features. In this work, we consider the area depleted of electrons to be a uniformly positively charged square. The confinement potential felt by electrons with charge, $-q_{0}$ $\left(q_{0}>0\right)$ in presence of a uniformly charged finite square (the depleted region) with length, $L$, and total positive charge, $Q$, is:

$$
U(x, y)=-q_{0} V(x, y)
$$

where $V(x, y)$ is the electrostatic potential created by a the uniformly charged square at the point $\vec{r}=(x, y)$ on the $2 \mathrm{D}$ plane. To calculate such a potential, we choose the origin of the system of coordinates at the center of the square. This implies that the positive charge is all found in the domain $D:-\frac{L}{2} \leq x^{\prime} \leq+\frac{L}{2} ;-\frac{L}{2} \leq y^{\prime} \leq+\frac{L}{2}$. The uniform charge density of such distribution is written as $\rho_{0}=Q / L^{2}$. The total charge of the domain, $Q$, and the length, $L$, can both be tuned experimentally. The calculation of the electrostatic potential, $V(x, y)$, is simplified using the transformation:

$$
\frac{1}{\left|\vec{r}-\vec{r}^{\prime}\right|}=\frac{2}{\sqrt{\pi}} \int_{0}^{\infty} d u e^{-u^{2}\left(\vec{r}-\vec{r}^{\prime}\right)^{2}} .
$$

The remaining steps are more straightforward with the final result for the potential written in integral form as:

$$
V(X, Y)=\frac{k Q}{L} \frac{2}{\sqrt{\pi}} \int_{0}^{\infty} d t f(t, X) f(t, Y),
$$

where $k$ is Coulomb's electric constant, $X=x / L, Y=$ $y / L$, and $t=u L$ are dimensionless variables. The function $f(t, X)$ (as well as $f(t, Y)$ ) is given by the following expression:

$$
f(t, X)=\frac{\sqrt{\pi}}{2 t}\left\{\operatorname{erf}\left[t\left(\frac{1}{2}-X\right)\right]+\operatorname{erf}\left[t\left(\frac{1}{2}+X\right)\right]\right\},
$$

where $\operatorname{erf}(z)=2 / \sqrt{\pi} \int_{0}^{z} d x e^{-x^{2}}$ is the error function [29]. The above expression is extremely useful since it allows us to calculate the electrostatic potential, thus the resulting confining energy, at any location on the $2 \mathrm{D}$ quantum-dot region. In dimensionless units, the confining potential, $U(X, Y)=-q_{0} V(X, Y)$, can be written as:

$$
U(X, Y)=-\frac{k Q q_{0}}{L} \frac{2}{\sqrt{\pi}} \int_{0}^{\infty} d t f(t, X) f(t, Y) .
$$

In Figure 1, we plot the confining potential as a function of the dimensionless distance, $\sqrt{X^{2}+Y^{2}}$, from the center of the square $(X=0, Y=0)$ for two chosen directions, $U(X, Y=0)$ and $U(X, Y=X)$, along the square. For the same distance from the center of the square, we verified that the strongest confinement is achieved along the

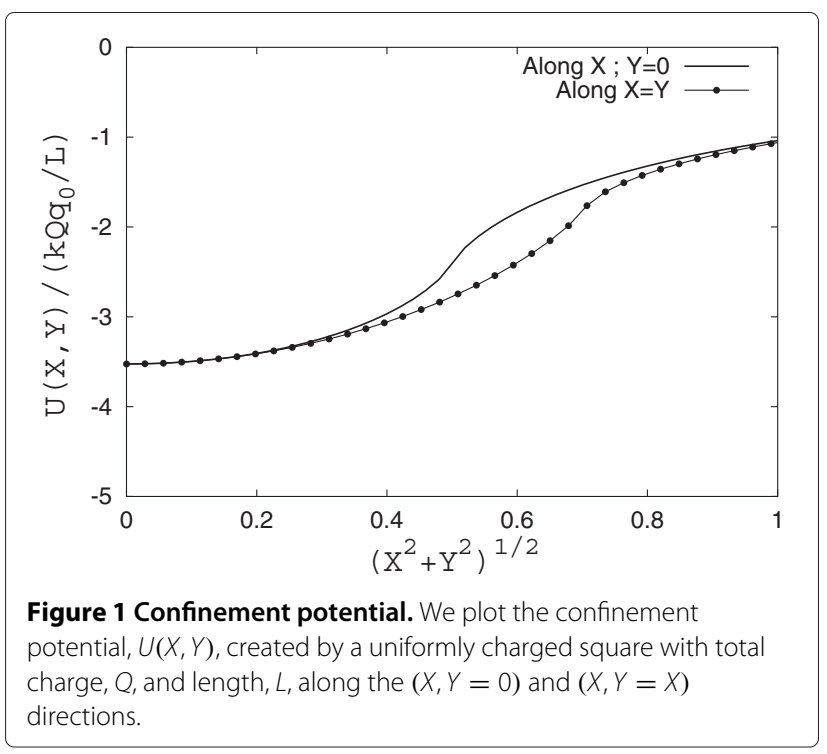


$Y=X$ direction. The overall strongest confinement energy felt by electrons was found to be:

$$
U(x=0, y=0) \approx-3.52549 \frac{k Q q_{0}}{L},
$$

and corresponds to electrons placed at the center of the uniformly charged square.

\section{Results and discussion}

\section{Approximated circular confinement potential}

The confinement potential created by a uniformly charged square is complicated given the dependence of the potential on each of the coordinates, $x$ and $y$, and not simply the distance, $\sqrt{x^{2}+y^{2}}$, from the center of the quantum dot. Thus, it looks very appealing to explore possibilities in which the realistic confinement potential for a squarepatterned quantum dot is replaced with an approximated circularly symmetric one as done in this work. To this effect, we assess the quality of such an approximation and also discuss instances in which one can reliably use the approximated simplified circular potential instead of the computationally unyielding exact one. To gain back the circular symmetry lost in the square, let us consider a circular domain in the form of a disk having the same area as the original square region:

$$
\pi R^{2}=L^{2}
$$

The idea is to compare the confinement potential created by a uniformly charged disk to the one created by a uniformly charged square under the constrain that the same total charge, $Q$, is uniformly distributed over two different domains with equal area. In this scenario, one has to calculate the resulting confining potential energy felt by an electron with charge, $-q_{0}\left(q_{0}>0\right)$, in a $2 \mathrm{D}$ uniformly charged disk. The calculation of such charged disk potential $[30,31]$ is lengthy with the final result:

$$
\begin{aligned}
& U_{D}(r, R)=-q_{0} V_{0} F(r, R), \\
& F(r, R)=\int_{0}^{\infty} \frac{d t}{t} J_{0}\left(\frac{r}{R} t\right) J_{1}(t),
\end{aligned}
$$

where $R$ is the radius of the disk, $F(r, R)$ is a function that depends only on the ratio $r / R$ (given in integral form), $J_{n}(x)$ are $n$-th order Bessel functions, $r=\sqrt{x^{2}+y^{2}}$ is the distance of the electron from the center of the disk, $V_{0}=(2 k Q) / R$ is the electrostatic potential created by the disk at its center $(r=0)$, and $k$ is Coulomb's electric constant. The uniformly charged circular-disk region with an area identical to the square is shown in Figure 2. The square domain with area $L^{2}=\pi R^{2}$ is shown in Figure 3 . Since the disk has the same area as the square, the radius of the disk is fixed to $R=L / \sqrt{\pi}$. As before, the most strongly

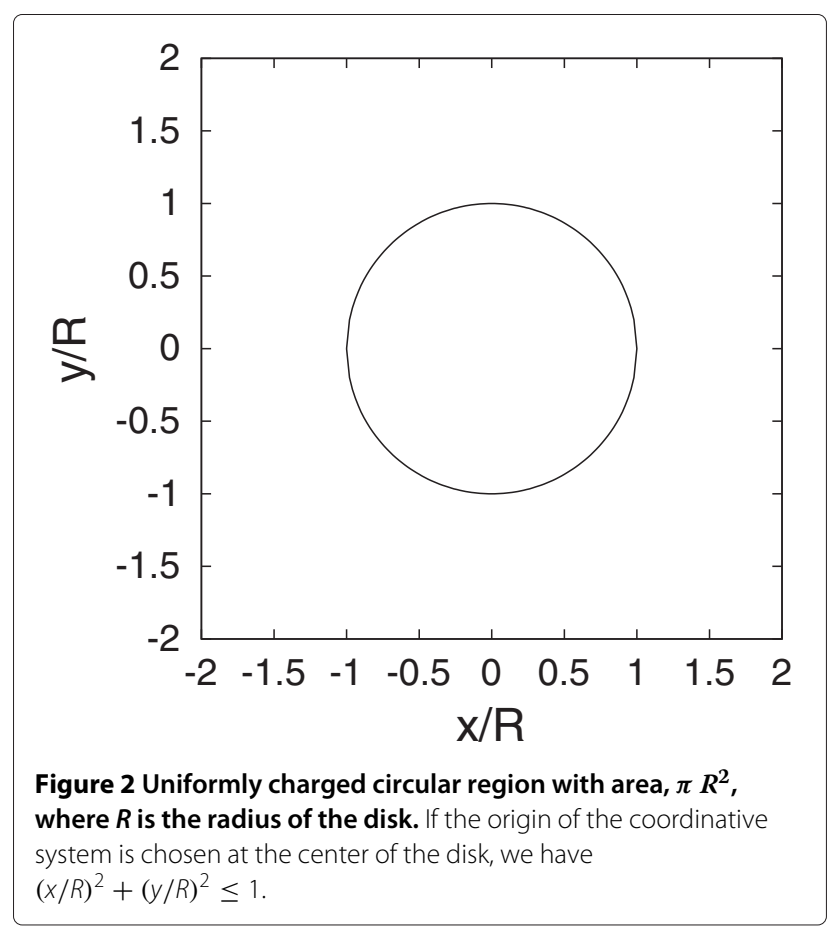

confined electrons are those localized at the center of the disk $(r=0)$ :

$$
\begin{aligned}
U_{D}\left(r=0, R=\frac{L}{\sqrt{\pi}}\right) & =-2 \frac{k Q q_{0}}{R} \\
& \approx-3.54491 \frac{k Q q_{0}}{L},
\end{aligned}
$$

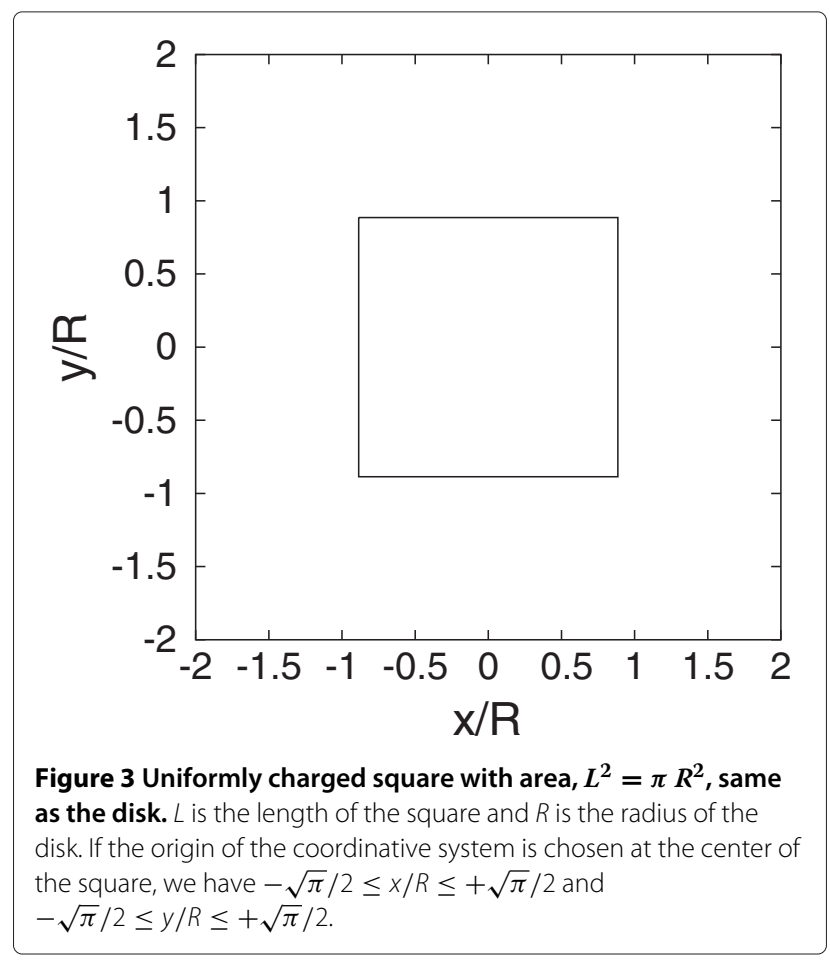


where the final value above was obtained after replacing $R=L / \sqrt{\pi}$. Compare the above approximated result in Equation (10) to the exact value, $U(x=0, y=0) \approx$ $-3.52549 k Q q_{0} / L$ from Equation (6). While such an initial result is encouraging, the overall quality of the approximation employed should be more thoroughly assessed by comparing the values of the exact confining potential, $U(x, y)$, at arbitrary points $(x, y)$ to the corresponding values obtained from the approximated circular confining potential, $U_{D}\left(r=\sqrt{x^{2}+y^{2}}, R=L / \sqrt{\pi}\right)$, at that location. For the sake of a direct comparison, as before, we measure the confinement energies in units of $k Q q_{0} / L$. After straightforward transformations, we rewrite:

$$
U_{D}(X, Y)=-\frac{k Q q_{0}}{L} 2 \sqrt{\pi} \int_{0}^{\infty} \frac{d t}{t} J_{0}\left(\sqrt{X^{2}+Y^{2}} \sqrt{\pi} t\right) J_{1}(t)
$$

where $X=x / L$ and $Y=y / L$ are the dimensionless coordinates suitable for the square case. In Figure 4, we plot the circular-disk confining potential, $U_{D}(X, Y)$, as a function of the dimensionless distance, $\sqrt{X^{2}+Y^{2}}$.

Based on simple arguments, we expect the approximated circular-disk potential to be a very good description of the exact non-circular potential in the central region of the domain and far away from the center of the domain. The most substantial differences are expected to occur at locations that correspond to edges such as sides of the square or corners of the square. We gauged the quality of $U_{D}(X, Y)$ by comparing it to the exact square potentials, $U(X, Y=0)$ and $U(X, Y=X)$, that represent the two boundary directions along the square's surface. The data clearly show that the agreement between the

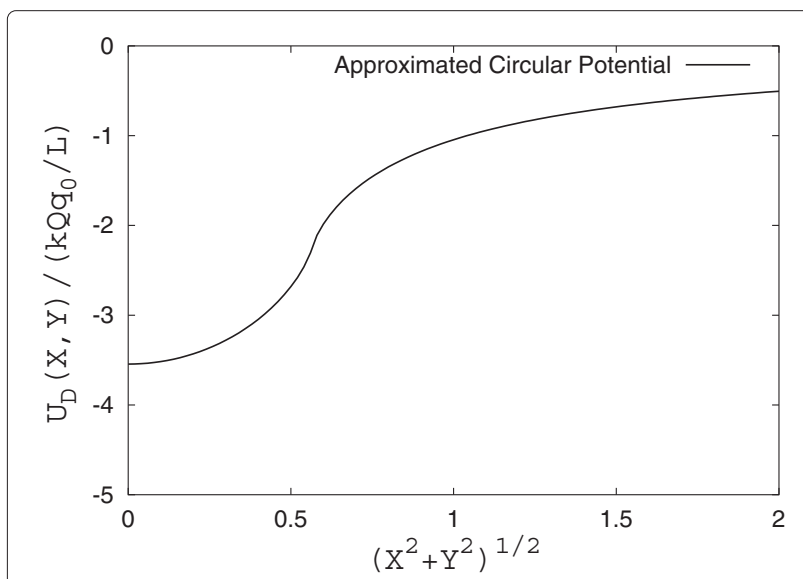

Figure 4 The circular confinement potential, $U_{D}(X, Y)$. This potential is created by a uniformly charged disk that approximates the exact non-circular potential originating from a square domain.

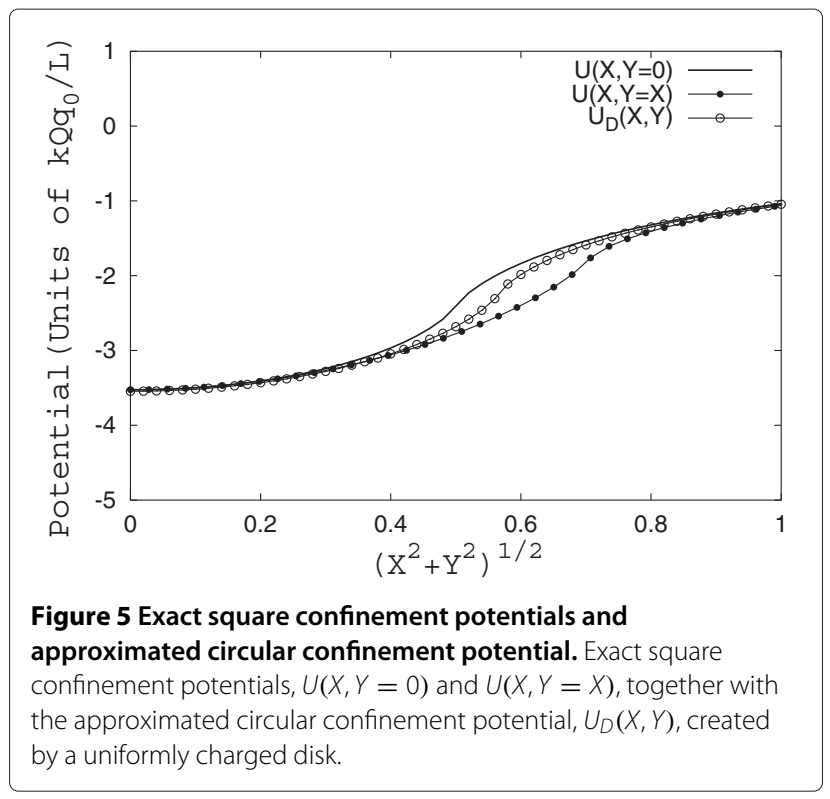

exact confinement potential and its approximated circular counterpart is very good everywhere with more pronounced variations at the edges of the domain. In Figure 5, we plot the realistic potentials, $U(X, Y=0)$ and $U(X, Y=$ $X)$, together with their approximated circularly symmetric disk confining potential, $U_{D}(X, Y)$.

\section{Conclusions}

The results indicate that the approximated circular symmetric potential is an excellent substitute for the realistic square potential almost everywhere, except the domain boundaries. Obviously, the particular analytic form of the realistic square potential, $U(x, y)$, is complicated given the dependence on both coordinates, rather than simply the distance from the center of the quantum dot. Thus, it is very appealing to find reliable substitutes for such a confinement that are simple and correctly capture key features. The approximated circular symmetric disk potential considered in this work appears to be a very reasonable choice for its simplicity and quality. Thus, we believe that the approximated simplified circular potential can be a reliable substitute for the computationally unyielding exact one for a square-patterned 2D semiconductor quantum dot in those cases where the speed of computational calculations, such as numerical diagonalizations [32] or other methods, is paramount.

Competing interests

The author declares that he has no competing interests.

Author's information

$\mathrm{OC}$ is an associate professor of Physics. His area of research is Theoretical Condensed Matter Physics. 


\section{Acknowledgements}

This research was supported in part by the National Science Foundation (NSF) under the grant number: DMR-1104795.

Received: 4 October 2012 Accepted: 31 October 2012

Published: 22 November 2012

\section{References}

1. Knoss, RW (ed.): Quantum Dots: Research, Technology and Applications Nova Science Publishers, New York (2008)

2. Jacak, L, Hawrylak, P, Wojs, A: Quantum Dots. Springer, New York (1998)

3. Michler, $P$ (ed.): Single Quantum Dots: Fundamentals, Applications and New Concepts. Springer, New York (2003)

4. Bird, JP (ed.): Electron Transport in Quantum Dots. Kluwer, Boston (2003)

5. Ciftja, O: Classical behavior of few-electron parabolic quantum dots. Physica B. 404, 1629 (2009)

6. Tavernier, MB, Anisimovas, E, Peeters, FM, Szafran, B, Adamowski, J, Bednarek, S: Four-electron quantum dot in a magnetic field. Phys. Rev. B. 68, 205305 (2003)

7. Ciftja, O, Faruk, MG: Two-dimensional quantum-dot helium in a magnetic field: variational theory. Phys. Rev. B. 72, 205334 (2005)

8. Pfannkuche, D, Gerhardts, RR, Maksym, PA, Gudmundsson, V: Theory of quantum dot helium. Physica B. 189, 6 (1993)

9. Kainz, J, Mikhailov, SA, Wensauer, A, Rössler, U: Quantum dots in high magnetic fields: calculation of ground-state properties. Phys. Rev. B. 65, $115305(2002)$

10. Ciftja, O: A Jastrow correlation factor for two-dimensional parabolic quantum dots. Mod. Phys. Lett. B. 23, 3055 (2009)

11. Merkt, U, Huser, J, Wagner, M: Energy spectra of two electrons in a harmonic quantum dot. Phys. Rev. B. 43,7320 (1991)

12. Tarucha, S, Austing, DG, Honda, T, van der Hage, RJ, Kouwenhoven, LP: Shell filling and spin effects in a few electron quantum dot. Phys. Rev. Lett. 77, 3613 (1996)

13. Sasaki, S, Austing, DG, Tarucha, S: Spin states in circular and elliptical quantum dots. Physica B. 256, 157 (1998)

14. Tsui, DC, Stormer, HL, Gossard, AC: Two-dimensional magnetotransport in the extreme quantum limit. Phys. Rev. Lett. 48, 1559 (1982)

15. Laughlin, RB: Anomalous quantum Hall effect: an incompressible quantum fluid with fractionally charged quasiparticles. Phys. Rev. Lett. 50, 1395 (1983)

16. Ciftja, O: Exact results for systems of electrons in the fractional quantum Hall regime II. Physica B. 404, 2244 (2009)

17. Ciftja, O: Exact results for systems of electrons in the fractional quantum Hall regime. Physica B. 404, 227 (2009)

18. MacDonald, AH, Johnson, MD: Magnetic oscillations of a fractional Hall dot. Phys. Rev. Lett. 70, 3107 (1993)

19. Ciftja, O: Effective hypernetted-chain study of even-denominator-filling state of the fractional quantum Hall effect. Phys. Rev. B. 59, 10194 (1999)

20. Kastner, MA: Artificial atoms. Phys. Today. 46(1), 24 (1993)

21. Ashoori, RC: Electrons in artificial atoms. Nature. 379, 413 (1996)

22. Kouwenhoven, LP, Marcus, CM: Quantum dots. Phys. World. 11, 35-39 (1998)

23. Ciftja, O: Generalized description of few-electron quantum dots at zero and nonzero magnetic field. J. Phys.: Condens. Matter. 19, 046220 (2007)

24. Taut, M: Two electrons in a homogeneous magnetic field: particular analytic solutions. J. Phys. A.: Math. Gen. 27, 1045 (1994)

25. Maksym, PA, Chakraborty, T: Quantum dots in a magnetic field: role of electron-electron interactions. Phys. Rev. Lett. 65, 108 (1990)

26. Yannouleas, C, Landman, U: Collective and independent-particle motion in two-electron artificial atoms. Phys. Rev. Lett. 85, 1726 (2000)

27. Adamowski, J, Sobkowicz, M, Szafran, B, Bednarek, S: Electron pair in a Gaussian confining potential. Phys. Rev. B. 62, 4234 (2000)

28. De Filippo, S, Salerno, M: Spectral properties of a model potential for quantum dots with smooth boundaries. Phys. Rev. B. 62, 4230 (2000)

29. Abramowitz, M, Stegun, IA: Handbook of Mathematical Functions, 9th ed. Dover, New York (1970)

30. Ciftja, O: An experimentally justified confining potential for electrons in two-dimensional semiconductor quantum dots. J. Comput. Aided Mater. Des. 14, 37 (2007)

31. Ciftja, O, Wexler, C: Monte Carlo simulation method for Laughlin-like states in a disk geometry. Phys. Rev. B. 67, 075304 (2003)
32. Ciftja, O, Faruk, MG: Two interacting electrons in a one-dimensional quantum-dot: exact numerical diagonalization. J. Phys.: Condens. Matter. 18, $2623(2006)$

doi:10.1186/2228-5326-2-36

Cite this article as: Ciftja: Realistic confinement potential for a squarepatterned two-dimensional semiconductor quantum dot and its approximated circular counterpart. International Nano Letters 2012 2:36.

\section{Submit your manuscript to a SpringerOpen ${ }^{\circ}$ journal and benefit from:}

- Convenient online submission

- Rigorous peer review

- Immediate publication on acceptance

- Open access: articles freely available online

- High visibility within the field

- Retaining the copyright to your article

Submit your next manuscript at $\boldsymbol{\nabla}$ springeropen.com 